\title{
Glioblastoma in a former Chernobyl resident 24 years later
}

\author{
Adam A. Dmytriw MSc, Gwynedd E. Pickett MD
}

Competing interests: None declared.

This article has been peer reviewed.

Correspondence to: Gwynedd E. Pickett, gwynedd.pickett@cdha. nshealth.ca

CMAJ 2013. DOI:10.1503 /cmaj.121660
1 previously healthy right-handed 39year-old man presented to the emergency department with a 5-month history of self-described personality changes, including anger issues. He had a 6-day history of expressive dysphasia, word substitution and slurring of speech. His medical history was notable only for smoking half a pack of cigarettes per day for 20 years. He had immigrated to Canada 10 years earlier, after living for a few years in Germany. However, he had been living in the Chernobyl greater municipal area, Ukraine, at the time of the Chernobyl disaster and for several years thereafter. Physical examination revealed difficulty with expressive speech. Computed tomography and magnetic resonance imaging of his brain showed a large, irregularly enhancing mass in the left posterior frontal lobe with surrounding edema and white matter infiltration. The appearance was consistent with a high-grade primary brain tumour in the region of the Broca area (Figure 1). Metastatic work-up, including positron emission scanning with fluorodeoxyglucose, was negative.

A left frontotemporal craniotomy was performed with debulking of the tumour. Glioblastoma multiforme (International classification of Disease for Oncology, code 9440/3; World Health Organization grade IV classification) was confirmed by pathology. Postoperative imaging showed good debulking with some deep residual tumour. Clinically, the patient continued to have some word-finding difficulties, but his diction improved. Radiation therapy was arranged; however, 3 weeks following surgery, progressive rightsided weakness and difficulty with speech developed, despite increasing doses of corticosteroids. Repeat imaging showed early and massive regrowth of the tumour, with the tumour appearing larger than on the initial preoperative scan.

At this point, the options for management included radiation therapy with concurrent and adjuvant temozolomide (chemoradiation), surgical debulking followed by chemoradiation, debulking with insertion of local chemotherapy (e.g., carmustine wafers) followed by chemoradiation, palliative radiation or referral for palliative care. Following extensive consultation with the multidisciplinary team, the patient underwent repeat debulking, followed by early radiation therapy. Repeat surgery was carried out without event. One week later, the patient underwent a short course of high-dose radiation therapy in combination with oral temozolomide. During the first week of radiation, his speech worsened further and his right-sided weakness progressed to the point that he could no longer walk. Computed tomography showed rapid regrowth of the tumour. Further active treatment with radiation and chemotherapy was discontinued, and palliative support was instituted. He died just over 4 months after initial presentation.

\section{Discussion}

The accident that occurred in April 1986 at the Chernobyl Nuclear Power Plant in Ukraine remains the worst accident at a nuclear power plant in history. ${ }^{1}$ Substantial radioactive fallout covered large parts of the western former Soviet Union. Until the nuclear accidents in Fukushima in 2011, the Chernobyl disaster was the only Level 7 event (i.e., major accident) on the International Nuclear Event Scale to have occurred.

Because of widespread exposure to ionizing radiation, there has been ongoing concern about 
the long-term consequences, particularly the risk of malignant disease, for those in the surrounding areas. An early study that examined exposed patients from 1986-1990 found an increased incidence of leukemia (incidence ratio 1.77, 95\% confidence interval [CI] 1.22-2.47) compared with the general population. ${ }^{1}$ Subsequent studies, which included follow-up to 1998, suggested an incidence ratio of 2.5 (90\% CI 1.3-3.7); however, the excess relative risk at 1 grey of 6.7 seen in this study was not statistically significant $(90 \%$ CI 0.8-23.5). ${ }^{1}$

Although there was no increased risk of thyroid cancer reported in studies performed 9 and 16 years after the Chernobyl accident, ${ }^{1}$ a later follow-up study that examined the same 16-year period with a much greater sample size showed an incidence ratio of 4.33 (95\% CI 3.29-5.60). ${ }^{1}$ In addition to a higher incidence of papillary thyroid malignancies among exposed people than among nonexposed people, the papillary tumours in exposed people were more likely to have an unusual solid morphology and c-RET oncogene rearrangement. This rearrangement has been associated with more rapid growth and a less-differentiated phenotype. ${ }^{1}$

Workers who cleaned up the Chernobyl reactor have been reported to be at greatest risk of thyroid cancer and leukemia. These were mainly men employed in 1986 and 1987, aged 20-45 years and who lived in the $30-\mathrm{km}$ exclusion zone. ${ }^{2}$ Young children were also at high risk. The relative risk of thyroid cancer was 237 (95\% CI 164-343) among those aged less than 1 year at the time of exposure; the relative risk was 6 (95\% CI 4-9) among 10-year-old children. ${ }^{3}$

It is difficult to quantify the risk of other solid tumours among those exposed by the Chernobyl accident, in part because the latency period for these cancers often exceeds the follow-up period of studies performed. ${ }^{2}$ One non-peer-reviewed report has suggested an increase in breast cancer among premenopausal women from contaminated areas of the Ukraine. ${ }^{2}$ An increased risk of glioma has been associated with highly penetrant, but extremely rare, genes and with ionizing radiation at high doses. ${ }^{4}$ Many products of these candidate genes likely interact with environmental factors; some studies suggest that irradiation is the only incontrovertible contributor to increased risk. ${ }^{5}$ Although few studies have explored glioma in the context of Chernobyl, there is one report of an increased incidence of oligoastrocytoma in Moldova. ${ }^{6}$

Ongoing and thorough data collection of malignant disease following nuclear accidents is necessary for an understanding of the long-term consequences of ionizing radiation. The lack of scientific consensus about the health effects of Chernobyl contributed to political and social pressures being brought to bear on medical diagnoses. ${ }^{7}$ Accurate and long-term documentation of health outcomes (ideally by global medical organizations, rather than politically defined entities) can help provide better estimates of population-based risk in the event of future nuclear events and can reduce legal and political disputes. Valid risk estimates do not relate only to conditions with increased risk: it is equally valuable to conclude, based on high-quality data, that the risk of a particular cancer or health condition is not increased.

\section{Latency period for malignant disease}

The initial impressions were that the radiation fallout would precipitate leukemia predominantly within 2 years of exposure; however, a

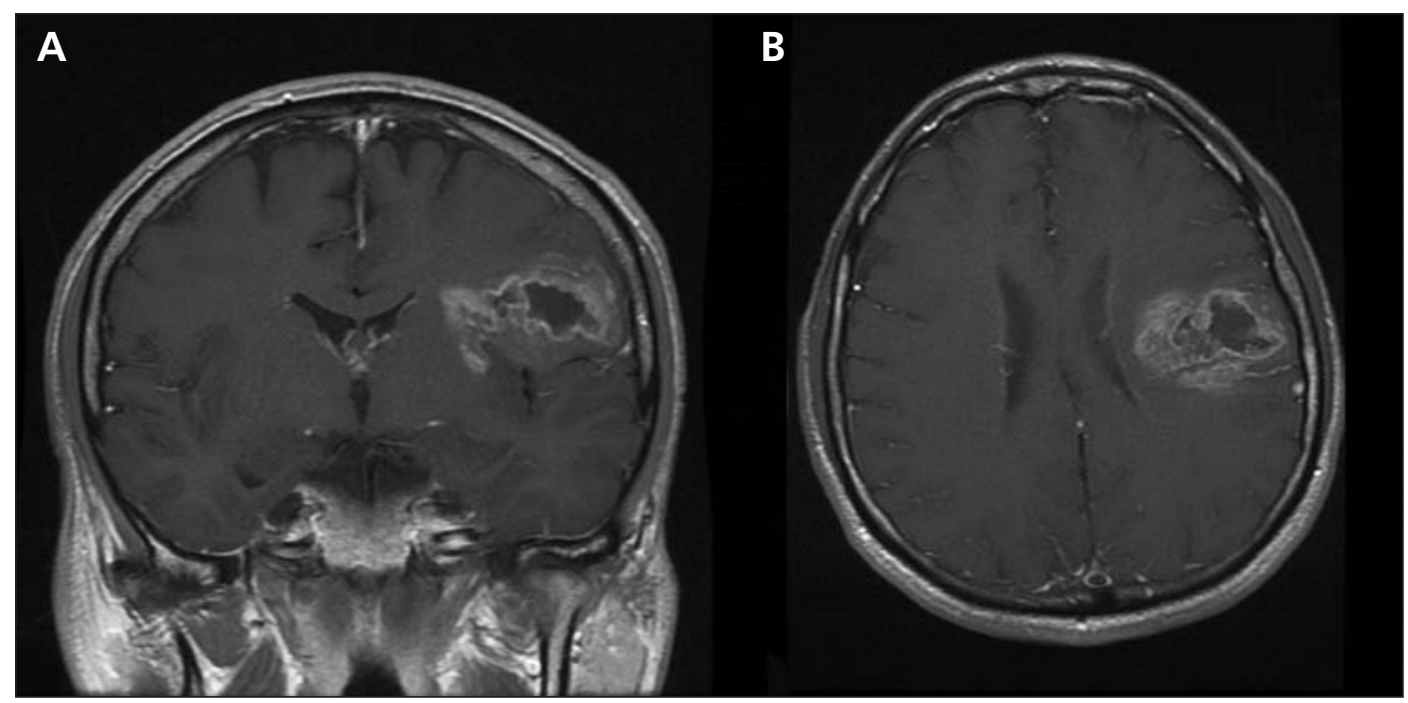

Figure 1: Coronal (A) and axial (B) T1-weighted magnetic resonance image, showing glioblastoma multiforme in the left hemisphere of a 39-year-old man. 
retrospective cohort study performed by the Russian Academy of Medical Sciences found that individuals who were within $30 \mathrm{~km}$ of the disaster may develop solid tumours, most notably thyroid malignancies, with a minimum latency period of 4 years. ${ }^{8}$ The United Nations Scientific Committee on the Effect of Atomic Radiation (UNSCEAR) originally projected a 10-year latency period. ${ }^{9}$ However, both reports clearly indicate that individuals who lived both inside the UNSCEAR "near zone" of fewer than $100 \mathrm{~km}$ and outside the UNSCEAR "far zone" of 100-2000 km may develop malignant disease beyond the minimum latency times normally associated with exposure to radiation. Particular concern has been expressed for the former residents of Pripyat and smaller Ukrainian villages in the Kiev provincial area that were not evacuated until days after the event.

In a 2002 conference of the United Nations Development Programme, oncologists expressed concern that a variety of solid tumours would emerge only decades after the incident. ${ }^{10}$ This concern was reinforced by the Chernobyl Research Unit of the National Institutes of Health's National Cancer Institute, which stated, "As the length of time since the accident increases to almost 20 years, there is interest in pursuing the lead with respect to breast cancer as well as in examining patterns with other solid tumors." ${ }^{2}$

\section{Registries}

The link between ionizing radiation and incident cancer is not new. However, to fully characterize the epidemiology, magnitude and nature of atomic risk on public health, particularly for tumours that may have very long latency, ongoing awareness and thorough data collection is required. In addition to maintaining a high index of suspicion for cancer in patients who may have been exposed to nuclear events in Chernobyl or Fukushima, it is important for physicians to report, with the patient's permission, any cases of cancer to the relevant international registries. Our patient, a Ukrainian immigrant to Canada who had previously lived in the affected area, presented with aggressive glioblastoma. We do not know with certainty whether his geographic history was a contributing cause, but given the

\section{Box 1: Cancer registries for major nuclear accidents}

- Cases of cancer among emigrants from Ukraine, Belarus or Russia should be reported to the Chernobyl Registry (Russia or Belarus: tel 748439933 90, info@nrer.ru; Ukraine: tel 3804425322 96, moz@mov.gov.ua)

- Cases of cancer among Japanese emigrants should be reported to the Fukushima Registry (tel 8124547 1412, ftiiki@fmu.ac.jp). rarity of this type of cancer and his young age, we believe that his exposure to the Chernobyl disaster may have contributed to the tumour's development. We sought and obtained permission to provide information on his diagnosis to the Chernobyl Registry (Box 1).

The Chernobyl Registry categorizes individuals by group (e.g., clean-up workers, evacuees, people living in the most-contaminated districts and their offspring). It seeks to follow the people included in the registry and is an important basis for future studies of the health of the exposed population. Practitioners should obtain patient consent to contribute the diagnoses and case details to the Chernobyl Registry upon seeing a former Ukrainian resident with cancer. Patients who emigrated from Belarus should also be considered at risk because of the drift of radioactive fallout, high radiation doses and the increased cancer incidence recorded in that area following the disaster.

Cases of cancer that arise among people who were living in Japan at the time of the Fukushima disaster should be reported to the Center for Cancer Control and Information Services at the Japan National Cancer Center or to the Fukushima Cancer Registry. Japan has considerable expertise in radiation-related issues, with decades of experience dealing with the consequences of radiation exposure in Hiroshima and Nagasaki. Japan rapidly instituted the Fukushima Basic Survey after the 2011 tsunami to ascertain exposure history. ${ }^{11}$ Fukushima Prefecture established a cancer registry in April 2010, before the disaster, and it will continue to record cancer cases in the district. However, there is difficulty in identifying cancer cases among members of the cohort who have moved away. ${ }^{11}$

It is important to avoid duplicate reporting of cases (e.g., cases that are captured in Canadian incidence statistics should not also be included in the same statistics in another country). Reporting only to local or national registries, however, can fail to provide a complete picture of the longterm consequences of a nuclear accident if the information about cancers among emigrants is not centrally collected. In this era of global mobility, international cooperation is essential. ${ }^{7}$

\section{Monitoring}

Screening for thyroid cancer has been suggested for people exposed to either the Chernobyl or Fukushima events. ${ }^{10,11}$ The United Nations Development Programme has also suggested screening for breast cancer among patients from the Chernobyl and Kievska regions. ${ }^{10}$ There is no indication at present to screen for additional types of cancer in either group. 


\section{Conclusion}

The long-term effects of exposure to nuclear disasters will remain underappreciated unless diligent reporting continues to be conducted in the future. Cases of cancer must be tracked among people who have emigrated from affected areas. These data should be centrally collected to ensure adequate capture and analysis. Canadian physicians should be aware that patients who immigrated from Ukraine, particularly from the Chernobyl area, are at risk of latent tumours, even 20 years or more after the event.

\section{References}

1. Williams D. Cancer after nuclear fallout: lessons from the Chernobyl accident. Nat Rev Cancer 2002;2:543-9.

2. Hatch M, Ron E, Bouville A, et al. The Chernobyl disaster: cancer following the accident at the Chernobyl nuclear power plant. Epidemiol Rev 2005; 27:56-66.

3. Thomas G, Karaoglou A, Williams ED. Radiation and thyroid cancer. Hackensack (NJ): World Scientific; 1999.

4. Fisher JL, Schwartzbaum JA, Wrensch M, et al. Epidemiology of brain tumors. Neurol Clin 2007; 25:867-90.

5. Schwartzbaum JA, Fisher JL, Aldape KD, et al.
Epidemiology and molecular pathology of glioma. Nat Clin Pract Neurol 2006;2:494-503.

6. Dumitrescu G, Logofatu S, Balan M, et al. The evolution of the incidence of glial brain tumors in Moldova in 1981-1991: the effect of excessive accidental hyper-radioactivity [article in Romanian]. Rev Med Chir Soc Med Nat Lasi 1995;99: 211-4.

7. Petryna A. Biological citizenship: the science and politics of Chernobyl-exposed populations. Osiris 2004; 19:250-65.

8. Cardis E, Howe G, Ron E, et al. Cancer consequences of the Chernobyl accident: 20 years on. $J$ Radiol Prot 2006;26:127-40.

9. Sources, effects and risks of ionizing radiation: Geneva (Switzerland): United Nations Scientific Committee on the Effects of Atomic Radiation; 1988.

10. The human consequences of the Chernobyl nuclear accident - a strategy for recovery. Geneva (Switzerland): United Nations Development Programme and UNICEF; 2002.

11. Akiba S. Epidemiological studies of Fukushima residents exposed to ionising radiation from the Fukushima Daiichi Nuclear Power Plant prefecture - a preliminary review of current plans. J Radiol Prot 2012;32:1-10.

Affiliations: Dalhousie Medical School (Dmytriw), Division of Neurosurgery; and the QEII Health S ciences Centre (Pickett), Halifax, NS.

Contributors: Gwynedd Pickett conceived the article. Adam Dmytriw reviewed the literature and drafted the manuscript. Gwynedd Pickett revised the manuscript for important intellectual content. Both of the authors approved the final version submitted for publication.
The section Cases presents brief case reports that convey clear, practical lessons. Preference is given to common presentations of important rare conditions, and important unusual presentations of common problems. Articles start with a case presentation (500 words maximum), and a discussion of the underlying condition follows (1000 words maximum). Visual elements (e.g., tables of the differential diagnosis, clinical features or diagnostic approach) are encouraged. Written consent from patients for publication of their story is a necessity and should accompany submissions. See information for authors at www.cmaj.ca.
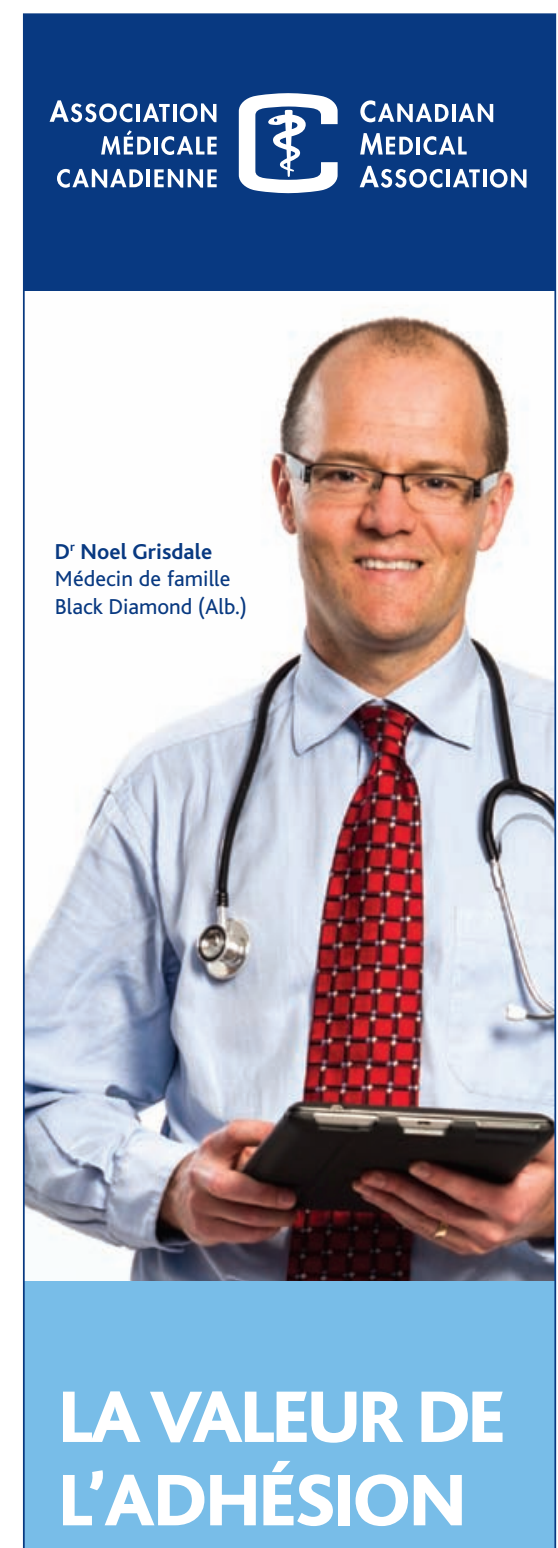

- Solutions de gestion globale du patrimoine

nessources et outils cliniques en ligne

- JAMC

- Représentation et promotion

Pour connaître tous les avantages de l'adhésion, composer le $\mathbf{8 8 8} \mathbf{8 5 5}$-2555 ou consulter amc.ca/membres 